\title{
The liver in siblings of patients with Indian childhood cirrhosis: a light and electron microscopic study
}

\author{
N C NAYAK, NEELAM MARWAHA, VEENA KALRA, SHEILA ROY, AND \\ O P GHA I
}

From the Departments of Pathology and Paediatrics, All India Institute of Medical Sciences, New Delhi, India

SUMMARY Liver biopsies from 29 siblings of patients with Indian childhood cirrhosis (ICC) and from two age-matched controls were examined by routine light and transmission electron microscopy. Histochemical stainings for copper and copper-binding protein were also carried out. The mild and non-specific structural alterations that were observed did not differ from those seen in control livers, even though a slight to moderate excess of copper and copper-binding protein was demonstrated in the majority. Aggregates of microtubules seen in some siblings, as well as in control livers, may indicate the preconditions for development of Mallory hyaline. It is possible that these features suggest a susceptibility for the development of ICC but not early disease.

Among several interesting features in the clinical and epidemiological spectrum of Indian childhood cirrhosis (ICC), there is one enigmatic one: the peculiar familial distribution of the disease. Even though no clear-cut pattern of inheritance is apparent, affliction of more than one child, twins, and other collateral members in the family has been described..$^{1-6}$ It has been suggested that an inherited susceptibility in children of the affected families may develop into frank disease under the precipitating influence of some unknown agent or agents. ${ }^{7}$ In the established stage of ICC, both clinical and pathological features appear to be characteristic. ${ }^{8}$ Hallmarks of earlier phases, however, remain to be clearly identified, though a precirrhotic symptom complex believed to herald the disease has been described. ${ }^{5}$ In a follow-up study of asymptomatic siblings, progression of early, non-specific hepatic alterations to classical changes of ICC was observed. ${ }^{7}$ Recently, considerable interest has centred around the finding of large excesses of copper and copper binding protein in the livers of patients with ICC..$^{9-11}$ The precise pathological effects of these materials in the development of hepatic lesions in this disease remain to be clarified. When we investigated a group of ICC patients and siblings of such patients to ascertain the part played by excessive hepatic copper and copperbinding protein in the evolution of the disease (Marwaha et al., submitted for publication), we took particular note of the structural alterations in the sibling liver, which are described in the present com-

Receiveo for publication 29 October 1980 munication. It was considered important from the point of view of the affected families to determine if there was any specific abnormality in the sibling livers and, because of ethical considerations, permission was obtained before performing the biopsies.

\section{Methods}

Percutaneous needle biopsy of the liver was obtained from 29 siblings of patients with Indian childhood cirrhosis (ICC); in 15 of the latter the diagnosis had been histologically confirmed. The age distribution was as follows: ICC patients-age range 10 months to 3 years (mean age 1.77 years), siblings of ICC patients-age range 2 months to 6 years (mean age 1.6 years). The major part of the biopsy specimen was fixed in formol saline and processed routinely for paraffin embedding followed by serial sectioning. Deparaffinised sections were stained with haematoxylin and eosin, modified Shikata's orcein stain ${ }^{12}$ for copper binding protein, p-dimethyl aminobenzylidene rhodanine stain for copper, and the periodic acid schiff (PAS) stain. A small portion of the liver biopsy specimen from 19 siblings was fixed in $3 \%$ glutaraldehyde, post-fixed in $1 \%$ osmium tetroxide, and processed in the conventional manner for transmission electron microscopy. Sections were examined with a Philips 300 electron microscope for specific organelle damage and for the presence of any abnormal structures within the hepatocyte. Wedge biopsy of the liver from two children who were operated on for congenital megacolon, but who had 


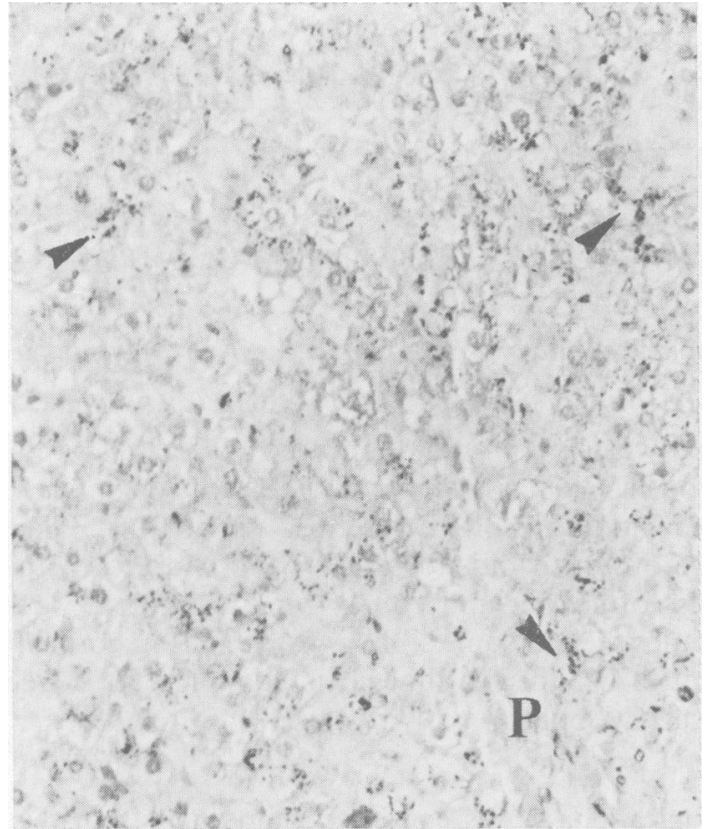

Fig. 1 Sibling liver showing moderate amounts of orcein positive deposits (arrow heads) in the periportal hepatocytes. $P$ : Portal tract. $\times 380$.

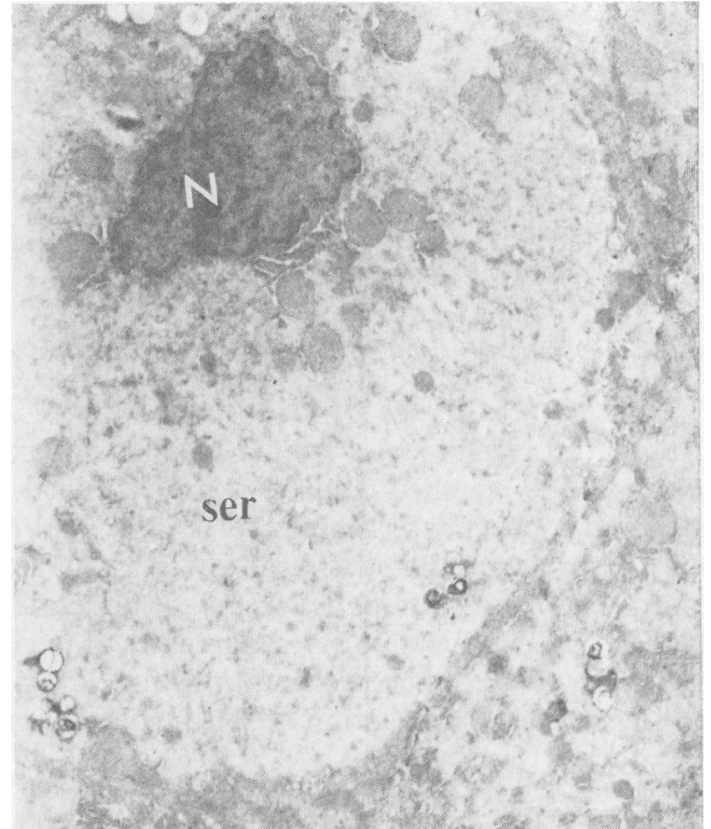

Fig. 2 Sibling liver showing irregular configuration of the nuclear membrane ( $N$ ) and numerous vesicles of smooth endoplasmic reticulum (ser) in the cytoplasm. $\times 6000$.

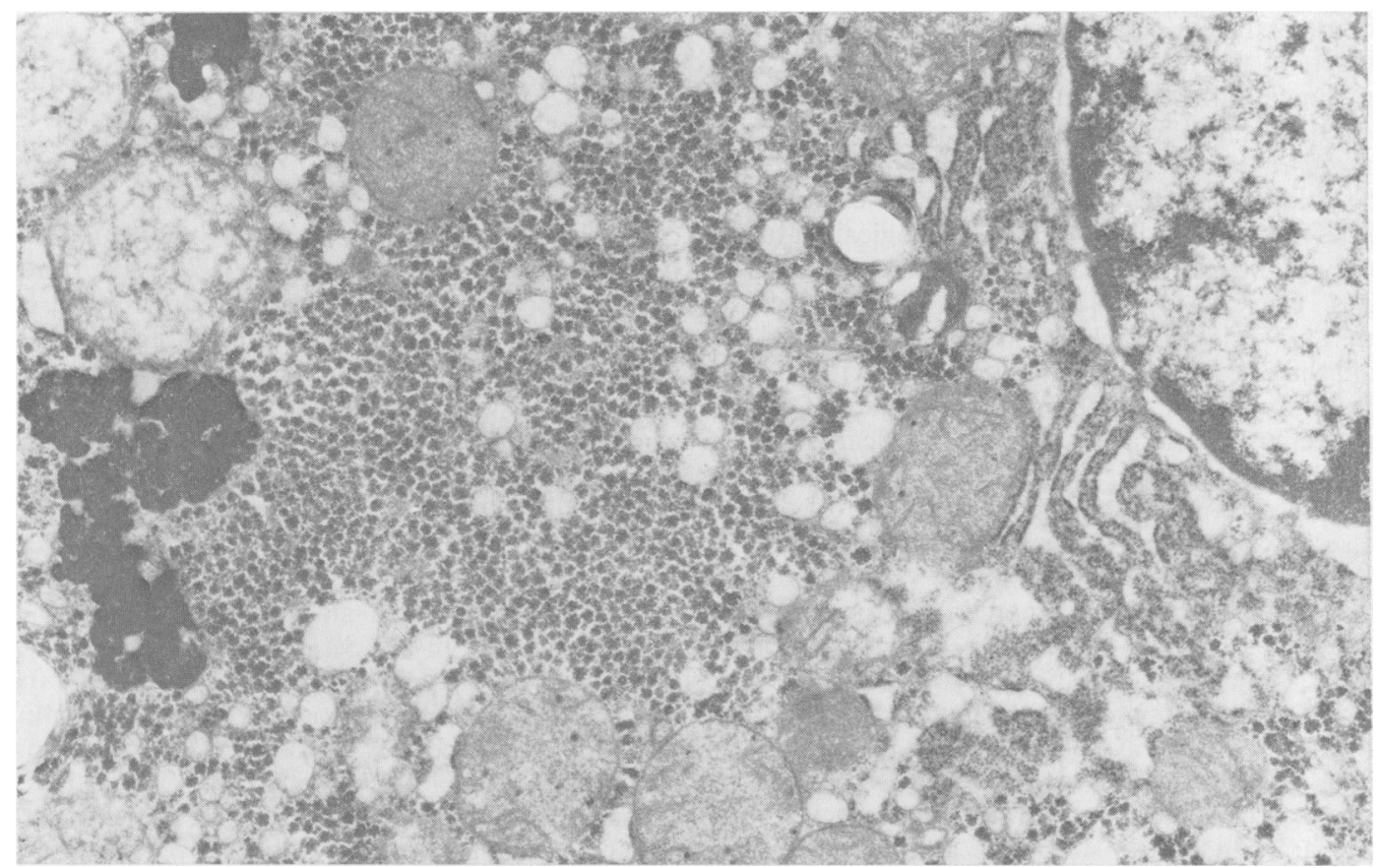

Fig. 3 Abundant glycogen rosettes of alpha particles, dilated sacs of smooth endoplasmic reticulum, and a few profiles of rough endoplasmic reticulum close to the nucleus. Lipofuscin pigment granules and two autophagic vacuoles are also seen. $\times 16400$. 


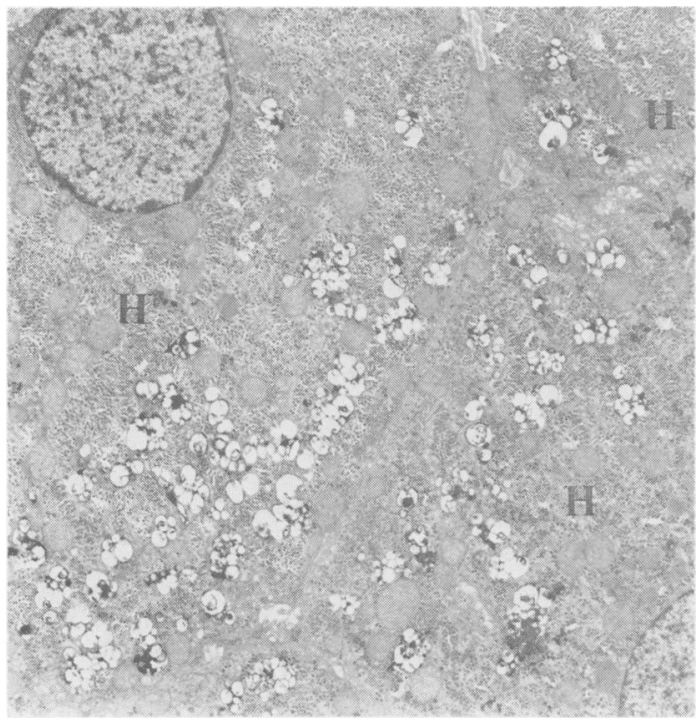

Fig. 4 Three adjacent hepatocytes $(H)$ show excess of lipofuscin pigment and numerous autophagic vacuoles. $\times 6600$. no clinical or biochemical evidence of liver disorder, served as control material. These were also processed for light and electron microscopy.

\section{Results}

LIGHT MICROSCOPIC STRUCTURE

Histological examination of the sibling livers revealed non-specific morphological features in the form of cytoplasmic and/or nuclear vacuolation in hepatocytes, and focal fatty change and portal fibrosis with or without the presence of a few mononuclear inflammatory cells in 16 out of 29 cases; the propositi of eight of these cases had been histologically proven. There appeared to be no difference in these minor abnormalities between siblings in whom the propositi had proved to have Indian childhood cirrhosis or those who had not. In the remaining cases the liver appeared to be normal by light microscopy. Copper was seen as orange red rhodanine positive intracytoplasmic granules, $0 \cdot 5$ $2 \mu$ in diameter, localised to the periportal hepatocytes in mild to moderate amounts in 18 out of 29 livers. Copper-binding protein, appearing as orcein-

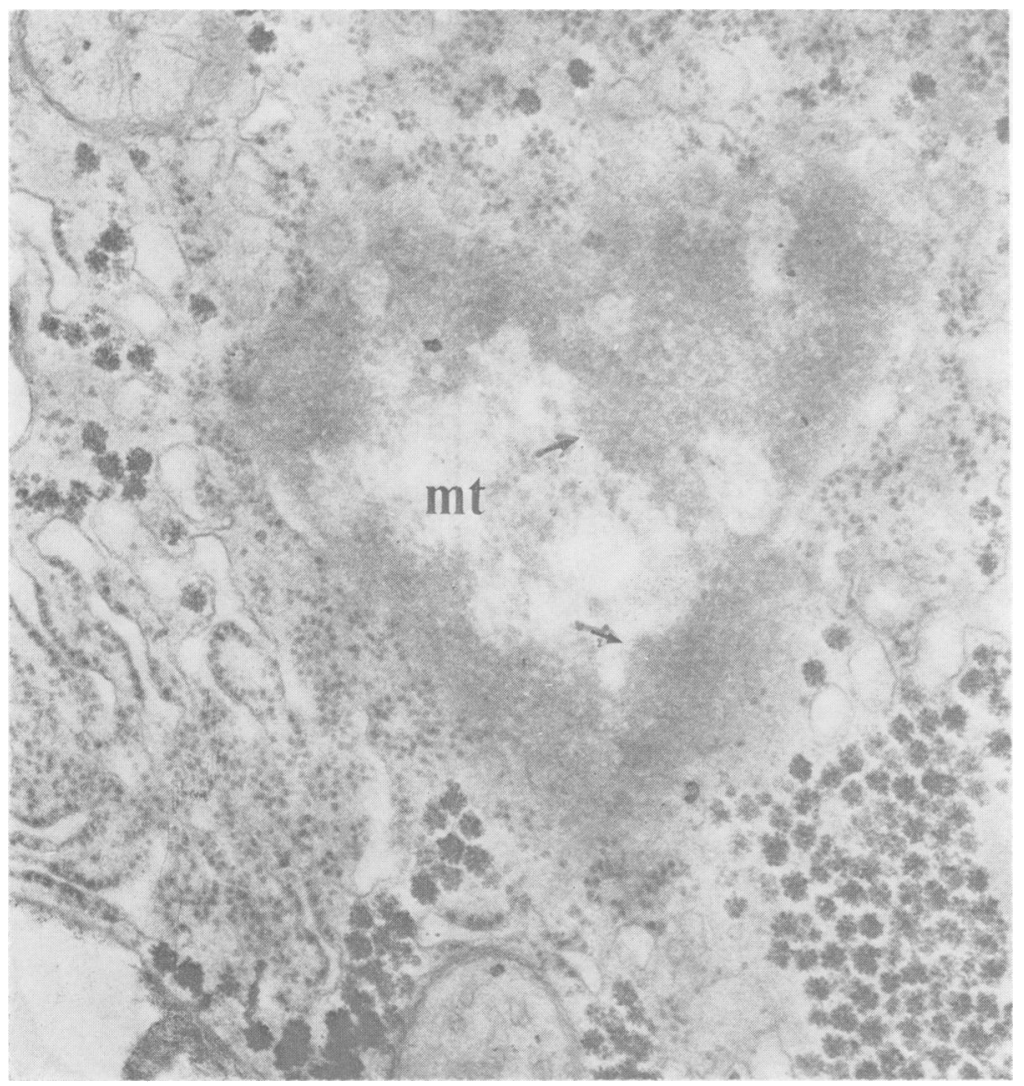

Fig. 5 An aggregate of microtubules (mt arrows) in proximity to polysomes and profiles of rough endoplasmic reticulum. $\times 20000$. 
positive, caramel coloured, coarse granules (Fig. 1), was present in 23 livers, its distribution and amount correlating well with those of copper. Non-specific histological alterations were seen in 12 out of 18 $(66.6 \%)$ of copper positive cases and in four out of $11(36.4 \%)$ of copper negative cases, the difference not being statistically significant. Also, no consistent histological abnormality could be observed in association with excess of tissue copper. The livers of two control cases were histologically unremarkable, except for mild excess of lipofuscin pigment in one. No copper or copper-binding protein was detected.

\section{ULTRASTR UCTURE}

The livers revealed a variable picture. In two biopsies the nuclear membranes of hepatocytes appeared to be irregular (Fig. 2), leading to loss of the normal rounded contour of the nucleus, but the chromatin and nucleolus seemed unremarkable. Moderate to abundant glycogen deposits were observed in the cytoplasm of hepatocytes in 14 siblings, displacing the mitochondria to the perinuclear area or to the cell membrane. Glycogen appeared as rosette-like aggregations of electron dense alpha particles (Fig. 3). In two biopsies relatively electron-lucent particles resembling the beta form of glycogen were scattered in some areas of the cytoplasm. Smooth endoplasmic reticulum (SER) was seen as groups of somewhat widened vesicles, diffusely scattered or interspersed among glycogen rosettes (Figs. 2 and 3). Rough endoplasmic reticulum (RER) was mainly located in the perimitochondrial and perinuclear regions and occasionally appeared to be dilated. In some hepatocytes focal aggregates of polysomes were seen in abundance. Mitochondria varied markedly in size and somewhat in shape, but their finer structure seemed to be undisturbed. Lipofuscin granules were conspicuous in seven sibling livers and, in two of these, prominent autophagic vacuoles were seen (Fig. 4). No zonal distribution of lipofuscin could be discerned. In seven biopsies occasional aggregates of microtubules were observed (Figs 5 and 6). These were random collections of haphazardly oriented microtubules ranging in diameter from $20-41 \mathrm{~nm}$. Some were in continuity with, while others were in proximity to, the RER profiles. An intermingled granular material was present in some of the microtubule aggregates.

Livers of eight siblings showed numerous irregular clear spaces devoid of any organelles or glycogen. Myelin figures and lipid vacuoles were prominent in a few biopsies and occasionally a widened intercellular junction or a dilated bile canaliculus was noted.

Hepatic ultrastructure in control livers was similar
Fig. 6 Details of microtubules (arrow) in an aggregate in the cytoplasm of $a$ hepatocyte. $\times 46000$.

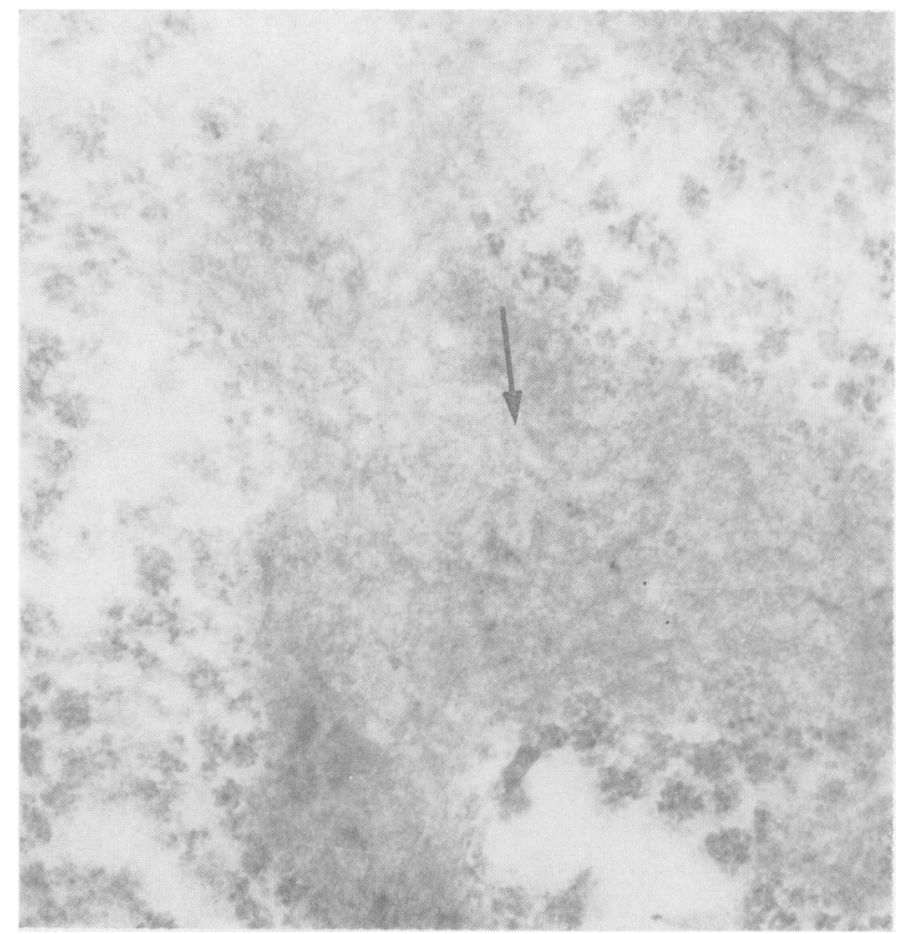


to that described in sibling livers including the presence of beta particles of glycogen in one liver. In one case moderately large collections of microtubules, morphologically identical to those seen in some of the sibling livers, were observed (Fig. 7).

\section{Discussion}

Hepatic morphology in siblings of ICC patients reveals no significant or consistent abnormality and the non-specific alterations seen in some do not correlate with the presence of mild to moderate excess of copper and copper-binding protein. Ultrastructural features of these livers do not differ from those of the two controls who had no apparent liver disease. In fact, although, for obvious reasons, information on hepatic ultrastructure in the healthy human is lacking, observations made in our subjects fall well within the spectrum of organelle morphology described by $\mathrm{Ma}$ and Biempica ${ }^{13}$ in biopsies from apparently normal livers obtained from patients with nonhepatic diseases who were subjected to different surgical procedures. We, therefore, believe that, in the asymptomatic siblings of those with
Indian childhood cirrhosis, hepatic morphology at best presents mild and non-specific alterations within the range of the so-called 'normal'. Moreover, a follow-up of 200 siblings over a period of six months to three years revealed not only that none progressed to ICC but that the hepatomegaly present in some regressed (Marwaha et al., submitted for publication).

An interesting finding in seven out of 19 sibling livers and in one of the two control livers was random aggregates of microtubules in the cytoplasm of liver cells- a feature not mentioned by $\mathrm{Ma}$ and Biempica ${ }^{13}$ in their report on the ultrastructure of the normal human liver cell. In the human liver occasional microtubules have been observed in the aggregates of microfilaments representing Mallory hyaline in various disorders including ICC. $^{\mathbf{1 4} 15}$ Random aggregates of microtubules have also been described in hepatocytes of alcoholics even in the absence of characteristic Mallory bodies..$^{15}$ Microtubules are formed by circumferential alignment of protofilaments constituted by tubulin-a protein synthesised by ribosomes. ${ }^{16}$ Tubulin monomers undergo polymerisation in the presence of a microtubule-associated protein to form dimers and oligo-

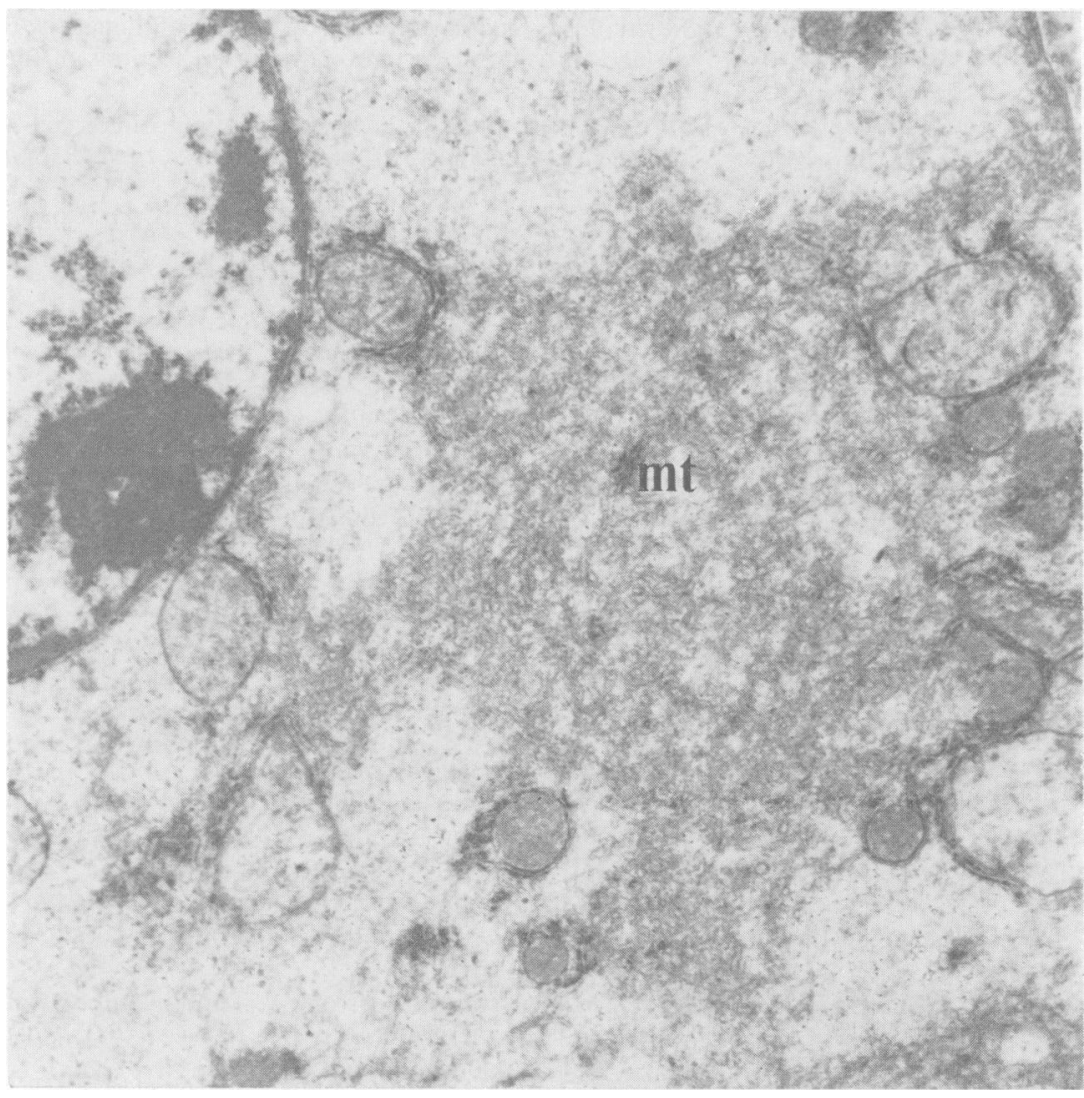

Fig. 7 Hepatocyte from a control liver showing a well defined aggregate of microtubules ( $\mathrm{mt}$ ) along with scattered beta particles of glycogen. $\times 12000$ 
mers which assume globular configurations and link up to form protofilaments. ${ }^{16}$ The exact mode of degradation of microtubules is not known, but this possibly occurs through formation of intermediatesized filaments which are $6-11 \mathrm{~nm}$ in diameter. ${ }^{17}$ Studies on experimental animals indicate that these intermediate filaments are likely precursors of Mallory hyaline, which is composed of aggregates of $20-27 \mathrm{~nm}$ diameter filaments. ${ }^{17-19}$ Griseofulvin administration to mice for a period of four to six weeks induces the formation of classical Mallory hyaline showing all three ultrastructural variants described in alcoholic liver disease ${ }^{20}$; and ICC. ${ }^{14}$ In in vitro cell culture systems low doses of griseofulvin applied for a few hours give rise to random 'broken' microtubule aggregates, while with higher doses given for a somewhat longer period of time collections of intermediate filaments are observed..$^{18}$ Thus microtubule disintegration may be an important step for Mallory hyaline induction, though the precise steps involved in its evolution are not clear. Priming and precipitating agents have been suggested, ${ }^{21}$ the nature of the former being unknown, while the latter are possibly anti-microtubule agents.

Development of Mallory hyaline is an important structural landmark in ICC. ${ }^{8}$ The finding of aggregates of microtubules in some of the sibling livers, therefore, assumes significance, even though no intermediate filaments or Mallory hyaline filaments were seen. Our studies on hepatic copper and copperbinding protein in children with ICC and their healthy siblings show that, in almost all of these subjects, a transient maturation defect of hepatocytes manifests itself in excess accumulation of these materials (Marwaha et al., submitted for publication) Copper in mild to moderate excess does not appear to be significantly injurious, but, once liver damage is initiated by other-as yet unknown-causes, copper may precipitate Mallory hyaline in an already 'primed' cell. Recent studies have shown that copper, by binding to sulfhydryl groups, can inhibit polymerisation of tubulin. ${ }^{22}$ The finding of collections of microtubules in some of the sibling as well as control livers may indicate some type of a 'priming' phenomenon. These changes probably resolve spontaneously unless a super-added injury results in progressive damage and Mallory hyaline formation.

\section{References}

${ }^{1}$ Singh A, Jolly SS, Kumar LR. Indian childhood cirrhosis. Lancet 1961; 1: 587-8.

${ }^{2}$ Agarwal RL. A comparative study of malnutrition and infantile cirrhosis of the liver. Arch Child Hlth 1961; 3: 6-11.
${ }^{3}$ Mohan M, Bhargava SK, Sobti JC, Taneja PN. Indian childhood cirrhosis-a heredofamilial disease. Indian Pediatr 1967 ;4: 125-30.

${ }^{4}$ Nayak NC, Visalakshi Singh M, Chawla V, Chandra RK, Ramalingaswami V. Indian childhood cirrhosis: a re-evaluation of its pathomorphologic features. Indian $J$ Med Res 1972; 60: 246-54.

${ }^{5}$ Parekh S, Patel BD. An epidemiologic survey of Indian childhood cirrhosis. Indian Pediatr 1972; 9: 431-9.

${ }^{6}$ Chawla V, Chandra RK, Verma IC, Ghai OP. An epidemiologic approach to Indian childhood cirrhosis. Indian Pediatr 1973; 10: 73-9.

'Patel BD, Parekh S, Chitale AR. Histopathological evolution of Indian childhood cirrhosis with emphasis on criteria of early diagnosis. Indian Pediatr 1974; 11 : 19-28.

${ }^{8}$ Nayak NC, Ramalingaswami V. Childhood cirrhosis. Clin Gastroenterol 1975; 4: 333-49.

${ }^{9}$ Portmann B, Mowat AP, Tanner MS, Williams R. Orcein positive liver deposits in Indian childhood cirrhosis. Lancet 1978; 1: 1338-40.

${ }^{10}$ Tanner MS, Portmann B, Mowat AP et al. Increased hepatic copper concentrations in Indian childhood cirrhosis. Lancet 1979; 2: 1203-5.

"Popper H, Goldfischer S, Sternlieb I, Nayak NC, Madhavan TV. Cytochemical copper and its toxic effects. Studies in Indian childhood cirrhosis. Lancet 1979; 2 : 1205-8.

${ }^{12}$ Nayak NC, Sachdeva R. Localisation of hepatitis B surface antigen in conventional paraffin sections of the liver. Am J Pathol 1975; 81: 479-85.

${ }^{13} \mathrm{Ma} \mathrm{MH}$, Biempica L. The normal human liver cellcytochemical and ultrastructural studies. Am J Pathol 1971; 62: 353-70.

${ }^{14}$ Nayak NC, Roy. Morphological types of hepatocellular hyalin in Indian childhood cirrhosis-an ultrastructural study. Gut 1976; 17: 791-6.

${ }^{15}$ Peterson P. Alcoholic hyalin, microfilaments and microtubules in alcoholic hepatitis. Acta Pathol Microl Scand 1977; Section A, 85: 384-94.

${ }^{16}$ Snyder AJ, McIntosh JR. Biochemistry and Physiology of microtubules. Ann Rev Biochem 1976; 45: 699-720.

${ }^{17}$ Sim JS, Frankes KE, French SW, Caldwel MG. Mallory bodies compared with microfilament hyperplasia. Arch Pathol Lab Med 1977; 101 : 401-11.

${ }^{18}$ Weber K, Wehland J, Herzog W. Griseofilavin acts with microtubules both in vivo and in vitro. J Molec Biol 1976; 102: 817-29.

${ }^{19}$ Franke W, Denk H, Schmid E, Osborn M, Klaus W. Ultrastructural biochemical and immunologic characterisation of mallory bodies in griseofulvin treated mice. Lab Invest 1979; 40: 201-20.

${ }^{20}$ Yokoo H, Minick OT, Batti F, Kent G. Morphologic varients of alcoholic hyalin. Am J Pathol 1972; 69: 25-33.

${ }^{21}$ Denk H, Eckerstorfer R. Colchicine induced mallory body formation in the mouse. Lab Invest 1977; 36: 353-5.

${ }^{22}$ Wallen M, Larsson H, Edstrom A. Tubulin sulphydryl groups and polymerisation in vitro. Exp Cell Res 1977; 107: 219-25. 